\title{
CROSTAS BIOLÓGICAS EM SAPROLITOS DE GNAISSE: CICLAGEM BIOGEOQUÍMICA, MICROMORFOLOGIA E ENSAIO DE COLONIZAÇÃO(1)
}

\author{
E. S. TRINDADE (2), C. E. G. R. SCHAEFE R(3), M. A. ALBUQUERQUE(4), \\ W. A. P. ABRAHÃO(3), J . W. V. MELLO ${ }^{(3)} \&$ A. C. CHAGAS(5)
}

\begin{abstract}
RESUMO
O papel biogeoquímico e estrutural das crostas microbióticas é praticamente desconhecido, embora estas sejam freqüentes na superfície de taludes naturais e antrópicos expostos em domínio tropical úmido. 0 presente trabalho teve como objetivo estudar as interações envolvidas no intemperismo biogeoquímico decorrente da ação de microrganismos e plantas inferiores na superfície de saprolitos gnáissicos expostos em taludes da Zona da Mata de Minas Gerais. Foram investigados também os efeitos das associações organominerais na formação e estabilização estrutural de agregados, bem como as feições micropedológicas das diferentes crostas e do substrato sob influência destas. Fez-se o isolamento de cianobactérias, objetivando uma visão preliminar da viabilidade de obtenção de inóculo em laboratório, para posterior utilização na estabilização de superfícies minerais expostas. 0 grau de intemperismo e o caráter máfico ou félsi co dos substratos foram determi nantes no comportamento dos organismos em relação à ciclagem biogeoquímica, influenciando os valores de $\mathrm{pH}$, a atividade de argila e o caráter eutrófico ou distrófico das crostas e saprolitos. Em geral, observou-se concentração de $\mathrm{K}$ e Al trocáveis na crosta, sendo $\mathrm{K}$ o elemento mais consistentemente associado à ciclagem biogeoquímica. O mesmo ocorreu em relação a Ca e Mg trocáveis, exceto nos saprolitos mais máficos, onde sua abundância mascarou a ciclagem biogeoquímica. As crostas tenderam também a concentrar $\mathrm{P}, \mathrm{Mn}, \mathrm{Pb}$ e Ni disponíveis, em todos os pontos, embora a contribuição de poluentes atmosféricos, no caso de $\mathbf{P b}$, possa estar mascarando os efeitos da atividade microbiana na mobilidade deste elemento.
\end{abstract}

\footnotetext{
(1) Trabalho extraído de Tese de Mestrado defendida como parte das exigências do Programa de Pós-Graduação em Sol os e Nutrição de Plantas da Universidade Federal de Viçosa - UFV. Recebido para publicação em agosto de 2000 e aprovado em junho de 2001.

(2) Doutoranda no Programa de Pós-Graduação em Solos e Nutrição de Plantas da U niversidade Federal deViçosa - UFV. CEP 36571000 Viçosa (MG). E-mail: trindade@solos.ufv.br

(3) Professor Adjunto do Departamento de Solos e Nutrição de Plantas, UFV. Bolsista do CNPq. E-mails: carlos.schaefer@sol os.ufv.br; wabrahao@mail.ufv.br; jwmello@solos.ufv.br

(4) Professora da Fundação Educacional de Caratinga - FU NEC. Av. Moacir de Mattos 49, Centro, CEP $35300-047$ Caratinga (MG).

(5) Estagiária Iniciação Científica do Departamento de Solos, UFV.
} 
Os teores de $\mathbf{N}$ disponíveis foram elevados, em decorrência do $\mathbf{N}$ fixado pelas cianobactérias. Os valores de Fe-ditionito, juntamente com os resultados das observações micropedológicas, mostraram um modelo de oxidação por influência microbiótica em microssítios da crosta e da camada micropedogenizada subjacente, baseado na liberação de $\mathrm{O}_{2}$ pelas cianobactérias e na utilização de quelatos de Fe-MO por bactérias quimiolitotróficas, que derivam energia da oxidação do Fe, promovendo a formação de micronódulos ferruginosos. Fotomicrografias obtidas em microscópio eletrônico de varredura ilustram, de modo inequívoco, o papel da mucilagem de polissacarídeos na estruturação de agregados, unindo a matéria orgânica fresca à parte mineral. Os resultados evidenciaram a possibilidade do uso de inóculo de algas na recuperação e estabilização de superfícies minerais expostas, por meio da aceleração do processo de sucessão ecológica.

Termos de indexação: crosta biológica, ciclagem biogeoquímica, micromorfologia, cultura de algas.

\section{SUMMARY: BIOTIC CRUSTS ON WEATHERED GNEISS: BIOGEOCHEMICAL CYCLING, MICROMORPHOLOGY AND EXPERIMENTAL COLONIZATION}

In the tropical humid environment, microbiotic crusts are widespread on exposed natural and antropic saprolites; yet, their biogeochemical and structural role is virtually ignored. In this work, theinteractions involved in the bi ogeochemi cal weathering resultant of the action by microorganisms and inferior plants in gneiss saprolites of the Zona da Mata of Minas Gerais region were studied. The effects of organo-minerals associations on the formation and structural stabilization of the aggregates were investigated as well as themicropedol ogical of features of thedifferent crusts and of thearea under their influence. In addition, cyanobacteria wereisolated in culturemedium, as themain organisms present in the microbiotic crusts, to evaluate the feasibility of their controlled inoculation in saprol ite colonization and stabilization. The weathering degree and mafic or felsic nature of the gneiss influenced the range of $\mathrm{pH}$ values, clay activity and the eutrophic/ dystrophic character of microbiotic crusts and saprolites. In general, $\mathrm{K}$ and $\mathrm{Al}$ tended to concentrate in the microbiotic crusts, together with $\mathrm{Ca}$ and $\mathrm{Mg}$, except for the mafic saprolites, where high $\mathrm{Ca} / \mathrm{Mg}$ reserves masked the biochemical cycling. Also, available $\mathrm{P}, \mathrm{Mn}, \mathrm{Pb}$ and $\mathrm{Ni}$ were concentrated in the crusts at all points, though a possible atmospheric contribution of $\mathrm{Pb}$ is likely. Available $\mathrm{N}$ levels were high in the microbiotic crusts due to $\mathrm{N}$ fixation by cyanobacteria. The Fediti onite combined with micropedological observations suggested a model of $\mathrm{Fe}$ oxidation mediated by microorganisms, based on excess $\mathrm{O}_{2}$ produced by cyanobacteria and further utilization of $\mathrm{Fe}$-organic matter by chemolithotrophic bacteria, that derive energy from Feoxidation, promoting widespread occurrence of ferruginous micronodules. TheSEM observations reveal ed a marked influence of mucilageon structural stabilization, bridging the organic matter/ mineral components. This points to a possible use of algae inoculation as a rehabilitation strategy in recently-exposed road-cuts.

Index terms: biol ogical crust, biogeochemical cyding, micromorphol ogy, culture of algae.

\section{NTRODUÇÃO}

As crostas biológicas, formadas por assembléias de microrganismos, dentre os quais bactérias, fungos, al gas elíquens, em associação com plantas inferiores, têm papel significativo no processo de agregação do solo, graças ao invól ucro das partículas minerais por tramas de filamentos e cimentantes orgânicos (polissacarídeos). Por esta razão, alguns trabalhos têm ressaltado sua importância na estabilização de sol os sobre os quais são esparsas ou ausentes plantas superiores (Ehrlich, 1996; Neuman et al., 1996; Pérez, 1997; Barker et al., 1998; Gorbushina \& Krumbein, 2000). No entanto, a importância das 
crostas biológicas na estabilização e recuperação de superfícies minerais é bem mais ampla. As comunidades componentes das crostas são pioneiras no processo de sucessão ecológica (Longton, 1992), mantendo a umidade do solo e disponibilizando elementos essenciais à instalação de plantas superiores por meio do intemperismo químico do substrato mineral, síntese de compostos orgânicos e fixação de N atmosférico (Zimmerman, 1993; Albrecht, 1999).

Os efeitos biológicos no intemperismo do solo envolvem a solubilização e precipitação de elementos, transformação de minerais primários e secundários, formação de depósitos e síntese de minerais (Robert \& Berthelin, 1986). A efetividade da dissolução de minerais por via bioquímica depende, em última análise, da interação de pH e natureza do ácido orgânico envolvido quanto à capacidade de complexação (disponibilidade de ligantes orgânicos extracelulares) esolubilidadeem água (Tan, 1980; Stone, 1998). Conforme Stone (1998), embora os mecanismos de formação de complexos organominerais tenham papel importante na dinâmica dos íons metálicos em ambientes naturais, seu entendimento é ainda bastante limitado.

Neste trabalho, foram analisados os efeitos da ciclagem biogeoquímica, associada à ocorrência de crostas biológicas, na mobilidade de macro, micronutrientes e metais pesados em saprolitos de gnaisse, expostos em taludes naturais e antrópicos. Foram investigados também os efeitos das associações organominerais na formação e estabilização estrutural de agregados, bem como as feições mi cropedológi cas das diferentes crostas e da área sob influência destas. Este ensaio em laboratório envolvendo o preparo de meios decultura para o desenvol vimento de al gas teve como objetivos não só melhor visual izar a diversidade biológica nas crostas estudadas, mas também avaliar a viabilidade de sua utilização na proteção de taludes expostos por meio da aceleração do processo natural de sucessão ecológica.

\section{MATERIAL E MÉTODOS}

\section{Descrição das áreas de amostragem}

Amostras representativas de superfícies colonizadas em saprolitos desenvolvidos de substratos gnáissicos foram coletadas em taludes naturais eantrópicos, na Zona da Mata do estado de Minas Gerais, entre as coordenadas 2040' e 21이오 S e 4230' e 43005' WGr. A região é marcada por clima úmido, apresentando verões quentes, coincidentes com a estação chuvosa, e invernos amenos, com curta estação seca. A pluviosidade média anual varia entre 1.200 e $1.500 \mathrm{~mm}$ e a temperatura, entre $21,8 \mathrm{e}$
19,5 느. Os principais litótipos regionais são representados por gnaisses bandados gerados em zona de cisalhamento de alto e baixo ângulo, migmatizados em graus variados, encontrados no domínio da Província Geotectônica Mantiqueira. A composição mineralógica desses litótipos mostra presença de plagiodásio cálcico, quartzo, biotita, anfibólio e muscovita em proporções variadas, além de traços de microclina, clorita, apatita, zircão, epidoto, titanita e granada (Brasil, 1991).

As col etas foram feitas através demicrotrincheiras, sendo discriminadas amostras da cobertura orgânica superficial, camada intermediária (aproximadamente 0-5 $\mathrm{mm}$ de profundidade) e saprolito (abaixo de $50 \mathrm{~mm}$ ).

\section{Análises físicas, químicas e mineralógicas}

Em laboratório, as amostras for am submetidas a análises físicas, sendo consideradas a densidade de partículas, densidade do solo, porosidade, textura e percentagem deagregados determinada por via seca (EMBRAPA, 1997). As análises químicas de rotina compreenderam a determinação de $\mathrm{pH}$ em água (relação 1:2,5), teores disponíveis de P, K, Fe, Zn, Mn, $\mathrm{Cu}$ (extrator M ehlich-1), teores trocáveis de $\mathrm{Ca}, \mathrm{Mg}$, $\mathrm{Al}$ (extrator $\mathrm{KCl} 1 \mathrm{~mol} \mathrm{~L}^{-1}$ ) eacidez potencial $(\mathrm{H}+\mathrm{Al}$ - extrator acetato de cálcio 0,5 mol L-1, pH 7,0). A disponibilidade de metais pesados ( $\mathrm{Cd}, \mathrm{Cr}, \mathrm{Pb}$ e Ni) foi determinada pelo método Mehlich-1 modificado (EMBRAPA, 1997). Para a extração de elementos totais, utilizou-se solução triácida $\left(\mathrm{HNO}_{3}, \mathrm{HClO}_{4} \mathrm{e}\right.$ HF), conforme EMBRAPA (1997).

Os teores deóxidos de ferro ealumínio cristalinos na fração argila das amostras referentes à camada intermediária ( $\simeq$ 0-5 $\mathrm{mm}$ ) foram determinados a partir de três extrações sucessivas com solução de citrato-ditionito (Mehra \& J ackson, 1960; Coffin, 1963), e os teores de óxidos amorfos, com oxalato de amônio (McKeague \& Day, 1966). As frações desses elementos ligadas à matéria orgânica do solo também foram determinadas por meio de extração com solução mista de $\mathrm{CuCl} 0,5 \mathrm{~mol} \mathrm{~L}^{-1}+\mathrm{KCl}$ 0,5 mol L-1 (Soon, 1993).

A determinação de nitrogênio total foi feita pelo método Kjeldahl de destilação a vapor (Bremner, 1996; Tedesco et al., 1985) e a de carbono total pela oxidação da matéria orgânica por via úmida, utilizando dicromato de potássio em meio sulfúrico (Nelson \& Sommers, 1982).

A fração argila da camada intermediária ( $~ 0$ $5 \mathrm{~mm}$ ) foi caracterizada mi neralogi camente por meio de difratometria de raio-X, tendo si do confeccionadas lâminas de argila natural e desferrificada. Foram considerados também, nessas últimas, tratamentos com KCl $1 \mathrm{~mol} \mathrm{~L}^{-1} ; \mathrm{MgCl}_{2} 1 \mathrm{~mol} \mathrm{~L}^{-1}$; $\mathrm{eMgCl}_{2}$ - glicerol (Whittig \& Allardice, 1986).

Para avaliar as feições micromorfológicas e pedogenéticas da crosta biológica e do saprolito 
adjacente, amostras indeformadas foram impregnadas com resina de poliéster que continha corante fluorescente (Uvitex OB; Ciba-Geigy), sendo confeccionadas lâminas delgadas no sentido transversal à crosta. As lâminas foram observadas em microscópio petrográfico (modelo OLYMPUS DX40) e suas feições micropedológicas descritas segundo Fitzpatrick (1993). As fotomicrografias foram tomadas com filme Kodacolor, $350 \mathrm{~mm}$, ISSO 100. Amostras indeformadas da crosta biológica foram também anal isadas em microscópio eletrônico de varredura (MEV) (modelo JEOLO 6400) para a visualização das interações organominerais no processo de formação de agregados. As fotografias foram feitas em filme Kodak, Verichrome pan, VPO 120, ISOO 125.

Amostras de crostas indeformadas foram também utilizadas na obtenção de culturas mistas de al gas, possibilitando uma melhor visualização da diversidade biológica na crosta, bem como a avaliação da viabilidade do preparo de inóculo em laboratório.

Em ambiente esterilizado, as crostas foram transferidas para erlenmeyers solução Bold Basal Medium(6) (Metting, 1994), para crescimento de microal gas (clorófitas, xantófitas e cianobactérias). O meio de cultura foi previamente autoclavado por 30 min., a uma temperatura de $121^{\circ} \mathrm{C}$, conforme procedimento descrito por Neuman et al. (1996).

Após três semanas, tendo sido observado um desenvolvimento expressivo de algas no meio de cultura, procedeu-se à repicagem do material. A segunda repicagem possibilitou o estabelecimento de uma cultura mista de microalgas, com ausência deresíduos minerais. Cada meio decultura foi então agitado separadamente e aspergido sobre blocos indeformados de saprolitos gnáissicos sem evidente col onização bi ol ógi ca, em duas repetições. Os blocos incubados foram manti dos em condições ambientes, em laboratório, sendo apenas protegidos por filme de PVC transparente. A umidade foi mantida pela aspersão regular da solução Bold Basal Medium sobre a superfície inoculada. U m bloco indeformado não inoculado foi mantido sob as mesmas condições dos blocos inoculados, servindo como controle.

Foram confeccionadas lâminas do material desenvol vido no meio de cultura, referenteà segunda repicagem. As lâminas foram examinadas em microscópio (OLYMPUS DX40), com câmera fotográfica acoplada.

\footnotetext{
(6) O meio líquido para o crescimento de microalgas é constituído por solução contendo macronutrientes $\left(\mathrm{NaNO}_{3}, \mathrm{~K}_{2} \mathrm{HPO}_{4}\right.$, $\mathrm{KH}_{2} \mathrm{PO}_{4}, \mathrm{MgSO}_{4} \cdot 7 \mathrm{H}_{2} \mathrm{O}, \mathrm{CaCl}_{2} \cdot 2 \mathrm{H}_{2} \mathrm{O}$ ), agentes quelantes $\left(\mathrm{Na}_{2}\right.$-EDTA, $\left.\mathrm{KOH}\right)$ e soluç̧ão de micronutrientes $\left(\mathrm{FeSO}_{4} \cdot 7 \mathrm{H}_{2} \mathrm{O}+\right.$ $\mathrm{H}_{2} \mathrm{SO}_{4}, \mathrm{H}_{3} \mathrm{BO}_{3}, \mathrm{MnCl}_{2} \cdot 4 \mathrm{H}_{2} \mathrm{O}, \mathrm{ZnSO}_{4} \cdot 7 \mathrm{H}_{2} \mathrm{O}, \mathrm{MoO}_{3}, \mathrm{CuSO}_{4} \cdot 5 \mathrm{H}_{2} \mathrm{O}$, $\left.\mathrm{Co}\left(\mathrm{NO}_{3}\right)_{2} \cdot 6 \mathrm{H}_{2} \mathrm{O}\right)$, a pH 6,6.
}

\section{RESULTADOS E DISCUSSÃO}

\section{Atributos físicos}

A transformação do saprolito fresco em camada micropedogenizada abaixo da crosta é evidenciada pela redução dos valores de silte e aumento do teor de argila (Quadro 1) em todos os pontos estudados (exceto em P2). Em geral, os teores de areia grossa e fina não mostraram tendência nítida de aumento ou redução, em virtude da heterogeneidade e alternância de bandas máficas efélsicas nos gnaisses estudados.

Com relaçãoàs classes deagregados determinados por via seca (Quadro 2), observou-se que os pontos que apresentaram maior incremento de argila na camada intermediária (P1, P3, P5 e P6) mostraram também maior percentagem deagregados nas faixas de diâmetro de 1,0-2,0 e 2,0-4,0 mm, onde a estruturação pedobiológica é mais evidente. A ação dos microrganismos na estruturação de agregados parece vinculada à abundante produção de mucilagem de polissacarídeos, que tende a unir a matéria orgânica fresca à parte mineral (Tisdall \& Oades, 1982; Oades, 1984). Nos sol os onde predominam agregados menores que $1,0 \mathrm{~mm}$, há grande contribuição da fração areia nos valores observados, o que mascara a real influência microbiótica na formação de agregados.

\section{Atributos químicos}

Os teores deK trocável (Quadro 3) foram sempre maiores na crosta, com fatores de concentração da ordem de 3,9 a 69,8 vezes, em relação aos teores observados no saprolito, diminuindo em termos absolutos nos saprol itos. No caso do Ca e Mg, ocorreu semel hante tendência, exceto em solo derivado de saprol ito quimi camente mais rico e máfico (P4 para Ca e P3 e P4 para Mg), onde os valores tenderam a aumentar em profundidade, mascarando os efeitos da ciclagem biogeoquímica na crosta. Os valores de P não foram consistentementeassociados à cidagem, tendo em vista a ineficiência do extrator ácido para formas orgânicas.

O Al trocável também aumentou do saprolito para a crosta em P2, P4, P5 e P6, independentemente do aumento dos teores de argila, indicando efetiva concentração biogênica e possível aumento da atividade microbiana para menores valores de $\mathrm{pH}$. Desse modo, a acidez orgânica e a oxidação de Fe, conforme discutido adiante, estariam provocando solubilização de Al, com parcial complexação pela matéria orgânica. Há poucos estudos sobre os efeitos tóxicos do Al em microrganismos.

Os teores de $\mathrm{N}$ nas crostas são el evados, possivel mente dada a abundância de cianobactérias, fixadoras de $\mathrm{N}$, em sua composição. Os valores verificados são comparáveis aos val ores normal mente encontrados no horizonte $A$ de solos sob vegetação 
Quadro 1. Caracterização física, segundo as faixas de amostragem nos diferentes substratos

\begin{tabular}{|c|c|c|c|c|c|}
\hline \multirow{2}{*}{ Substrato } & \multicolumn{4}{|c|}{ Textura } & \multirow{2}{*}{ Porosidade total } \\
\hline & Areia grossa & Areia fina & Silte & Argila & \\
\hline & 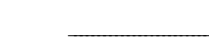 & -0 & & - & \\
\hline \multicolumn{6}{|l|}{ Ponto 1} \\
\hline $\begin{array}{l}\text { Camada intermediária } \\
\text { Saprolito }\end{array}$ & $\begin{array}{l}25,66 \\
25,66\end{array}$ & $\begin{array}{l}15,75 \\
15,94\end{array}$ & $\begin{array}{l}31,64 \\
37,05\end{array}$ & $\begin{array}{l}26,95 \\
21,36\end{array}$ & $\begin{array}{l}51,45 \\
50,93\end{array}$ \\
\hline \multicolumn{6}{|l|}{ Ponto 2} \\
\hline $\begin{array}{l}\text { Camada intermediária } \\
\text { Saprolito }\end{array}$ & $\begin{array}{l}50,79 \\
41,29\end{array}$ & $\begin{array}{l}25,02 \\
28,72\end{array}$ & $\begin{array}{l}14,49 \\
19,08\end{array}$ & $\begin{array}{r}9,70 \\
10,90\end{array}$ & $\begin{array}{l}52,20 \\
51,99\end{array}$ \\
\hline \multicolumn{6}{|l|}{ Ponto 3} \\
\hline $\begin{array}{l}\text { Camada intermediária } \\
\text { Saprolito }\end{array}$ & $\begin{array}{l}42,83 \\
49,97\end{array}$ & $\begin{array}{l}24,35 \\
22,43\end{array}$ & $\begin{array}{l}17,97 \\
18,30\end{array}$ & $\begin{array}{r}14,85 \\
9,30\end{array}$ & $\begin{array}{l}51,10 \\
48,74\end{array}$ \\
\hline \multicolumn{6}{|l|}{ Ponto 4} \\
\hline $\begin{array}{l}\text { Camada intermediária } \\
\text { Saprolito }\end{array}$ & $\begin{array}{l}70,78 \\
81,73\end{array}$ & $\begin{array}{l}26,93 \\
16,17\end{array}$ & $\begin{array}{l}1,89 \\
2,10\end{array}$ & $\begin{array}{l}0,50 \\
0,00\end{array}$ & $\begin{array}{l}48,27 \\
47,97\end{array}$ \\
\hline \multicolumn{6}{|l|}{ Ponto 5} \\
\hline $\begin{array}{l}\text { Camada intermediária } \\
\text { Saprolito }\end{array}$ & $\begin{array}{l}18,25 \\
23,54\end{array}$ & $\begin{array}{l}35,80 \\
33,80\end{array}$ & $\begin{array}{l}33,57 \\
42,56\end{array}$ & $\begin{array}{r}12,38 \\
0,09\end{array}$ & $\begin{array}{l}57,73 \\
57,65\end{array}$ \\
\hline \multicolumn{6}{|l|}{ Ponto 6} \\
\hline $\begin{array}{l}\text { Camada intermediária } \\
\text { Saprolito }\end{array}$ & $\begin{array}{l}40,90 \\
41,92\end{array}$ & $\begin{array}{l}22,66 \\
22,99\end{array}$ & $\begin{array}{l}28,99 \\
34,62\end{array}$ & $\begin{array}{l}7,45 \\
0,46\end{array}$ & $\begin{array}{l}49,93 \\
49,67\end{array}$ \\
\hline
\end{tabular}

Quadro 2. Percentagem de agregados, determinada por via seca, referente à camada intermediária $(\sim 0-5 \mathrm{~mm})$

\begin{tabular}{|c|c|c|c|c|c|c|}
\hline \multirow{2}{*}{ Substrato } & \multicolumn{6}{|c|}{ Diâmetro de agregados } \\
\hline & $>4 \mathrm{~mm}$ & $4,0-2,0 \mathrm{~mm}$ & $2,-0-1,0 \mathrm{~mm}$ & $1,0-0,5 \mathrm{~mm}$ & $0,5-0,25 \mathrm{~mm}$ & $<0,25 \mathrm{~mm}$ \\
\hline & & & & & & - \\
\hline Ponto 1 & 0,56 & 9,76 & 30,49 & 26,50 & 14,57 & 18,11 \\
\hline Ponto 2 & 1,00 & 4,02 & 7,55 & 23,90 & 29,31 & 34,22 \\
\hline Ponto 3 & 3,14 & 7,51 & 11,44 & 12,22 & 25,68 & 40,02 \\
\hline Ponto 4 & 1,64 & 2,98 & 4,88 & 20,43 & 32,14 & 37,93 \\
\hline Ponto 5 & 0,57 & 7,29 & 11,09 & 11,24 & 19,44 & 50,38 \\
\hline Ponto 6 & 0,13 & 2,70 & 13,11 & 20,51 & 23,77 & 39,77 \\
\hline
\end{tabular}

de mata, o que demonstra a importância das algas fixadoras de $\mathrm{N}$ na ciclagem biogeoquímica em materiais inci pientemente pedogenizados. Em geral, a relação $\mathrm{C} / \mathrm{N}$ apresenta-se equilibrada na crosta orgânica e bastante alta em subsuperfície, com val ores crescentes em profundidade, podendo indicar o baixo grau de humificação do material orgânico solúvel.

Os val ores de $\mathrm{H}+\mathrm{Al}$ acompanharam o aumento do carbono em superfície, mas, em P6, onde a crosta microbiana era mais espessa e dissociada do substrato mineral subjacente, os valores de $\mathrm{H}+\mathrm{Al}$ foram baixos em relação ao carbono orgânico total (Quadro 3), que alcançou 23,5\%. Os val ores da CTC total foram elevados nos pontos P3 e P4, quimicamente mais ricos e máficos que os demais, com aumento generalizado no sentido saprolitocamadas superficiais. Isto evidencia a contribuição dos colóides orgâni cos na geração de cargas, mesmo em crostas microbióticas delgadas. Apenas nesses 
Quadro 3. Caracterização química de rotina dos diferentes substratos, segundo as faixas de amostragem

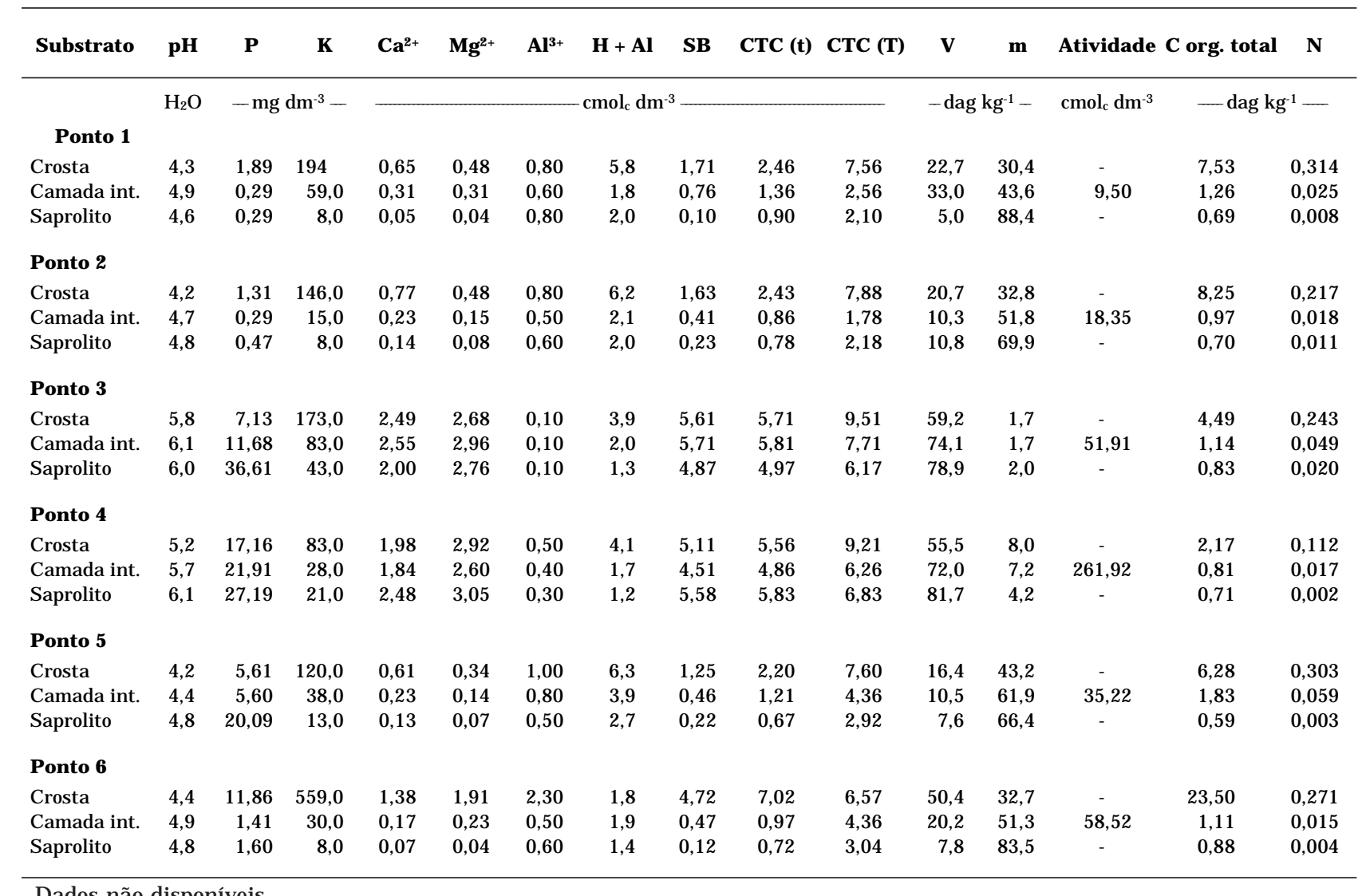

- Dados não-disponíveis.

perfis a saturação de bases foi elevada (>50\%), embora os val ores tenham diminuído em superfície, aumentando, em contrapartida, os val ores deH +Al. Esse fato ilustra a importância da pedogênese mesmo em escalas micrométricas, em substratos expostos à ação intempérica.

De maneira geral, os saprolitos de P1, P2, P5 e P6 apresentaram caráter álico, enquanto P3 e P4 foram caracterizados como eutróficos. Nesse sentido, pode-se, em princípio, separar as crostas em dois grupos distintos pelos atributos químicos, grau de intemperismoe, ou, maficidade do material parental. Os saprolitos em P3 e P4, situados em relevo escarpado, condicionado a fal hamentos introduzidos no Cinturão de Cisalhamento Atlântico (Artur \& Wernick, 1993), correspondem a áreas instáveis do ponto de vista geomorfológico, o que implica a exposição de materiais frescos à superfície e, portanto, a existência de condições de maior riqueza química. Os demais substratos ocorrem em áreas de maior estabilidade morfoestrutural, tendo sido submetidos a acentuado pré-intemperismo. Essa interpretação é corroborada pelos valores de atividade de argila calculados para a camada intermediária.
Quanto aos teores de micronutrientes e metais pesados (Quadro 4), observa-se que os teores de Fe, $\mathrm{Mn}$ e $\mathrm{Zn}$ disponível foram bem superiores nos saprolitos e crostas desenvolvidos de materiais quimi camente mais ricos e máficos (P3 eP4), embora os teores na crosta sejam inferiores aos teores verificados no saprolito. Os teores na crosta dependem da ciclagem biogeoquímica e, portanto, de fatores, tais como disponibilidade de água, luz e espécies de microrganismos presentes. Os valores de $\mathrm{Cu}$ e $\mathrm{Pb}$ não acompanharam as tendências de aumento em P3 e P4 verificadas para outros elementos, ao contrário, os valores de $\mathrm{Pb}$ foram menores nestes pontos, em relação aos demais. Sobretudo no que se refere ao P2, os teores de $\mathrm{Pb}$ podem ter sofrido influência de aportes de descarga de veículos, uma vez que os teores foram negligenciáveis em P4, único ponto onde não havia trânsito de veículos, considerando a inexistência de acesso por estrada. Em todos os pontos, os teores de Cu foram maiores na camada intermediária, o que pode estar refletindo uma menor mobilidade do elemento no ambiente, associada à constante incorporação bioquímica (residual) na camada intermediária e renovação orgânica na crosta. Nas 
Quadro 4. Microelementos e metais pesados disponíveis, segundo as faixas de amostragem nos diferentes substratos estudados

\begin{tabular}{|c|c|c|c|c|c|c|c|c|}
\hline Substrato & $\mathbf{F e}$ & Mn & Zn & $\mathrm{Cu}$ & Cd & $\mathbf{C r}$ & $\mathbf{P b}$ & $\mathbf{N i}$ \\
\hline & & & & $-r$ & 3 & & & - \\
\hline \multicolumn{9}{|l|}{ Ponto 1} \\
\hline Crosta & 53,4 & 15,1 & 1,33 & 0,20 & $\mathrm{Nd}$ & $\mathrm{Nd}$ & 0,17 & 0,023 \\
\hline Camada intermediária & 32,9 & 8,0 & 1,98 & 3,05 & $\mathrm{Nd}$ & $\mathrm{Nd}$ & 0,05 & $\mathrm{Nd}$ \\
\hline Saprolito & 31,2 & 0,8 & 0,08 & 0,12 & $\mathrm{Nd}$ & $\mathrm{Nd}$ & 0,09 & $\mathrm{Nd}$ \\
\hline \multicolumn{9}{|l|}{ Ponto 2} \\
\hline Crosta & 55,9 & 71,9 & 1,44 & 1,05 & $\mathrm{Nd}$ & $\mathrm{Nd}$ & 0,74 & 0,004 \\
\hline Camada intermediária & 32,6 & 17,0 & 2,34 & 4,36 & $\mathrm{Nd}$ & $\mathrm{Nd}$ & 0,21 & $\mathrm{Nd}$ \\
\hline Saprolito & 38,1 & 5,1 & 0,10 & 0,11 & $\mathrm{Nd}$ & $\mathrm{Nd}$ & 0,20 & $\mathrm{Nd}$ \\
\hline \multicolumn{9}{|l|}{ Ponto 3} \\
\hline Crosta & 67,3 & 102,9 & 1,61 & 1,01 & $\mathrm{Nd}$ & $\mathrm{Nd}$ & 0,10 & 0,022 \\
\hline Camada intermediária & 44,0 & 50,6 & 1,24 & 2,22 & $\mathrm{Nd}$ & $\mathrm{Nd}$ & 0,01 & 0,009 \\
\hline Saprolito & 55,8 & 16,3 & 0,52 & 0,55 & $\mathrm{Nd}$ & $\mathrm{Nd}$ & 0,05 & $\mathrm{Nd}$ \\
\hline \multicolumn{9}{|l|}{ Ponto 4} \\
\hline Crosta & 175,1 & 79,4 & 3,75 & 0,38 & $\mathrm{Nd}$ & $\mathrm{Nd}$ & 0,01 & 0,037 \\
\hline Camada intermediária & 164,8 & 38,7 & 7,85 & 3,64 & $\mathrm{Nd}$ & $\mathrm{Nd}$ & $\mathrm{Nd}$ & 0,023 \\
\hline Saprolito & 115,2 & 9,7 & 3,36 & 0,10 & $\mathrm{Nd}$ & $\mathrm{Nd}$ & 0,01 & $\mathrm{Nd}$ \\
\hline \multicolumn{9}{|l|}{ Ponto 5} \\
\hline Crosta & 129,8 & 109,6 & 2,76 & 0,47 & $\mathrm{Nd}$ & $\mathrm{Nd}$ & 0,26 & 0,068 \\
\hline Camada intermediária & 63,1 & 101,2 & 4,37 & 4,01 & $\mathrm{Nd}$ & $\mathrm{Nd}$ & 0,14 & 0,027 \\
\hline Saprolito & 28,8 & 6,8 & 0,19 & 0,09 & $\mathrm{Nd}$ & $\mathrm{Nd}$ & 0,13 & $\mathrm{Nd}$ \\
\hline \multicolumn{9}{|l|}{ Ponto 6} \\
\hline Crosta & 37,6 & 54,2 & 2,23 & 0,12 & $\mathrm{Nd}$ & $\mathrm{Nd}$ & 0,31 & 0,016 \\
\hline Camada intermediária & 20,9 & 13,2 & 1,98 & 2,47 & $\mathrm{Nd}$ & $\mathrm{Nd}$ & 0,17 & 0,025 \\
\hline Saprolito & 17,6 & 0,8 & 0,14 & 0,10 & $\mathrm{Nd}$ & $\mathrm{Nd}$ & 0,09 & $\mathrm{Nd}$ \\
\hline
\end{tabular}

crostas estudadas, não foram detectados teores mensuráveis de $\mathrm{Cd}$ e $\mathrm{Cr}$ disponíveis.

\section{Teores totais}

Os teores totais de $\mathrm{K}$ e Al (Quadro 5) foram, de maneira geral, fortemente influenciados pelo grau de intemperismo do saprolito. Os resultados obtidos são corroborados pela análise mineralógica do substratomineral por difratometria deraio- $X$, a qual revelou predomínio de ilitas e esmectitas (2:1) nos pontos $\mathrm{P} 3$ e P4, onde os teores de K eAl tenderam a reduzir-se em superfície. Por outro lado, a presença de vermiculita com hidróxi-intercamada-VHE e gi bbsita denota a natureza pré-intemperizada deP1, P2, P5 e P6, condições em que a ciclagem biogeoquímica de $\mathrm{K}$ eAl, com tendência à concentração na superfície microbiótica, torna-se mais evidente.

Também quanto aos teores totais, a concentração de Mg e Ca na superfície microbiótica variou conforme a constituição mineralógica do substrato gnáissico, com tendência à redução em superfícienos substratos de caráter máfico.
De maneira geral, os teores totais de Fe e $\mathrm{Mn}$ foram influenciados tanto pel ograu de intemperismo quanto pela maficidade do material de origem. Nas lâminas de micromorfologia, as quais serão discutidas a seguir, são nítidas as ocorrências de nódulos ferruginosos na transição crosta/camada mi cropedogenizada, o que explica a concentração de Fe nas camadas superficiais de P2, P3 e P5. Tendo em vista a maior mobilidade do $\mathrm{Mn}$ em relação ao $\mathrm{Fe}$, os teores deste el emento tenderam a valores mais el evados em direção às camadas superficiais de $\mathrm{P} 1$, P3 e P5, concentrando-se nas crostas de P2 e P6.

Apenas em P4, onde se observaram o caráter máfico e a menor percentagem de matéria orgânica na composição da crosta, os teores de $\mathrm{Mn}$ foram maiores em profundidade.

Os teores de Cu e Zn foram variáveis entre os pontos amostrados, apresentando, em geral, valores maiores onde os saprolitos apresentaram caráter máfico e em profundidade, exceto para Cu em P4. Para todos os pontos amostrados, observou-se uma tendência de redução do teor de Cd do saprol ito para 
Quadro 5. Teores totais de macro, micronutrientes e metais pesados, segundo as faixas de amostragem nos diferentes substratos

\begin{tabular}{|c|c|c|c|c|c|c|c|c|c|c|c|c|c|}
\hline Substrato & $\mathbf{P}$ & K & $\mathrm{Ca}$ & Mg & Al & $\mathbf{F e}$ & Mn & Zn & $\mathrm{Cu}$ & Cd & $\mathrm{Cr}$ & $\mathbf{P b}$ & $\mathbf{N i}$ \\
\hline & & & & & & $-m g d$ & $m^{-3}$ & & & & & & \\
\hline \multicolumn{14}{|l|}{ Ponto 1} \\
\hline Crosta & - & 854,5 & 474,6 & 804,5 & 92261,8 & 52237,2 & 112,4 & 38,9 & 18,4 & 2,8 & 11,9 & 19,5 & 50,1 \\
\hline Camada int. & - & 940,1 & 1614,9 & 86 & 7247 & 51416,7 & 143,0 & 40,0 & 27,8 & 1,5 & 117,4 & 14,3 & 55,2 \\
\hline Saprolito & - & 1419,9 & 338,6 & 107,6 & 98200,4 & 59556,3 & 92,6 & 42,4 & 20,1 & 0,2 & 99,7 & 11,0 & 55,0 \\
\hline \multicolumn{14}{|l|}{ Ponto 2} \\
\hline Crosta & - & 320,1 & 595,5 & 711,9 & 69139,5 & 42832,6 & 638,6 & 41,3 & 50,4 & 1,6 & 40,3 & 148,1 & 47,0 \\
\hline Cama & - & 62,4 & 1277,5 & & & 27943,0 & 103,5 & 47,2 & & 0,7 & 12,0 & 67,3 & 49,4 \\
\hline Saprolito & - & 30,9 & 1072,3 & 618,4 & 58213,0 & 37228,5 & 62,3 & 71,5 & 37,7 & 2,7 & 32,1 & 71,1 & 55,5 \\
\hline \multicolumn{14}{|l|}{ Ponto 3} \\
\hline Crosta & - & 11900,1 & 468,8 & 2023,9 & 61432,0 & 61150,4 & 684,4 & 77,1 & 41,6 & 1,6 & 94,2 & 40,5 & 66,2 \\
\hline Camada int. & & 26632,5 & 177,0 & 3194,4 & 63520,4 & 52910,1 & 597,5 & 91,5 & 51,8 & 1,3 & 91,5 & 47,6 & 91,2 \\
\hline Saprolito & - & 27777,9 & 165,9 & 3438,0 & 66386,8 & 49524,0 & 426,4 & 108,8 & 56,2 & 3,3 & 96,0 & 49,9 & 103,5 \\
\hline \multicolumn{14}{|l|}{ Ponto 4} \\
\hline Crosta & - & 22500,2 & 2033,2 & 2024,8 & 48531,6 & 43992,8 & 557,2 & 81,1 & 24,0 & 1,5 & 42,0 & 17,2 & 28,4 \\
\hline Cama & & 24537,5 & 2212,1 & 2934, & 57149,4 & 52773 & 790,0 & 113,2 & & 0,1 & 36 & 21,7 & 36,7 \\
\hline Saprolito & - & 29489,6 & 3677,9 & 3052,9 & 41222,4 & 59808,3 & 873,3 & 118,5 & 21,0 & 2,7 & 66,9 & 18,2 & 46,7 \\
\hline \multicolumn{14}{|l|}{ Ponto 5} \\
\hline Crosta & - & 1745,3 & 722,4 & 1798,7 & 65336,3 & 106248,8 & 1123,6 & 92,3 & 58,0 & 1,1 & 113,7 & 34,0 & 85,8 \\
\hline Camada in & & 1461,8 & 246,6 & 1321,7 & 58357,0 & 89690,6 & 1438,1 & 82,2 & 64 & 0,5 & 97 & 53,3 & 75,0 \\
\hline Saprolito & - & 1063,0 & 863,2 & 1120,9 & 67893,7 & 100991,6 & 1034,4 & 85,0 & 64,7 & 3,1 & 85,7 & 54,7 & 94,2 \\
\hline \multicolumn{14}{|l|}{ Ponto 6} \\
\hline Crosta & - & 1121,8 & 1346,6 & 1244,0 & 59524,1 & 25238,2 & 123,6 & 11,1 & 16,0 & 0,7 & 14,2 & 32,7 & 15,4 \\
\hline Camad & - & 397,4 & 349,0 & 622,9 & 88636,0 & 33996,4 & 39,3 & 9,2 & 19 , & 0,1 & 9,9 & 23,1 & 24,2 \\
\hline Saprolito & - & 415,5 & 96,1 & 202,2 & 86286,1 & 32040,1 & 13,4 & 18,0 & 16,2 & 2,2 & 10,6 & 14,8 & 24,0 \\
\hline
\end{tabular}

- Dados não-disponíveis.

a camada micropedogenizada de até 27 vezes (P4) e de concentração na crosta microbiótica. Os teores foram baixos em todos os pontos, não sofrendo variação significativa entre eles.

Da mesma forma, foi observada concentração de $\mathrm{Cr}$ na camada orgânica de todos os pontos amostrados, com valores elevados em P1 e P5 (>100 $\mathrm{mg} \mathrm{dm}^{-3}$ ). Ao contrário, os teores de $\mathrm{Ni}$ tenderam, de maneira geral, a valores maiores em profundidade. Os elementos $\mathrm{Cr}$ e $\mathrm{Ni}$ não apresentaram relação direta com a maficidade dos saprolitos, contrariamente ao esperado.

Os teores de $\mathrm{Pb}$ foram variáveis entre os pontos amostrados, tendendo a concentrar-se na camada orgânica de P1, P2, e P6. Os teores observados em P2 foram significativamente mais altos que nos demais pontos, atingindo $148,1 \mathrm{mg} \mathrm{dm}^{-3}$ na camada orgânica. Neste ponto, oteor dePb na crosta foi duas vezes maior que o teor verificado na camada mi cropedogenizada subjacente. Como observado na discussão dos teores disponíveis, é provável queestes teores estejam sendo incrementados pela contaminação de $\mathrm{Pb}$ proveniente da descarga de veículos, tendo em vista que os menores teores de $\mathrm{Pb}$ foram observados em $\mathrm{P} 4$, único ponto onde não havia trânsito de veículos na vizinhança.

\section{Frações de Fe e Al na camada micropedo- genizada}

Os teores de Fe amorfo (Quadro 6), extraído com oxalato $\left(\mathrm{Fe}_{\mathrm{o}}\right)$, foram negligíveis em P1, P2, P4 e P6, e bastante baixos em P3 e P5, o que evidencia a ocorrência de um microambiente favorável à oxidação do Fe em formas de mel hor cristalinidade.

Os el evados valores de $\mathrm{Fe}_{\mathrm{D}}$, juntamente com os resultados das observações micropedológicas, discutidos a seguir, revelam um papel relevante da atividade microbiótica na formação de nódulos hematíticos em microssítios da crosta e da camada micropedogenizada subjacente.

Em condições de boa aeração e pH entre 4 e 5, existe a possibilidade de utilização de quelatos de Fe-matéria orgânica por bactérias quimiorganotróficas 
(Kullman \& Schweisfurth, 1978, citado por Silver et al., 1986). Tais bactérias usam o carbono orgânico do ligante como fonte de energia, liberando $\mathrm{Fe}^{3+} \mathrm{em}$ soluções bem próximas da neutralidade.

Por outro lado, bactérias quimiolitotróficas, em meio ácido, podem utilizar a energia liberada na oxidação do $\mathrm{Fe}^{2+}$ em seu metabolismo, através da reaçãol:

Reação I. $2 \mathrm{Fe}^{2+}+1 / 2 \mathrm{O}_{2}+2 \mathrm{H}^{+} \rightarrow 2 \mathrm{Fe}^{3+}+\mathrm{H}_{2} \mathrm{O}$ $(\Delta \mathrm{G}=-6,5 \mathrm{kcal} / \mathrm{mol})$

Reação II. $2 \mathrm{Fe}^{3+}+6 \mathrm{H}_{2} \mathrm{O} \rightarrow 2 \mathrm{Fe}(\mathrm{OH})_{3}+6 \mathrm{H}$

A precipitação subseqüente do $\mathrm{Fe}^{3+}$ (reação II) libera prótons $\left(\mathrm{H}^{+}\right)$, acidificando o meio, o que pode resultar na desestabilização dos aluminossilicatos, com conseqüente liberação de Al trocável (Shen et al., 1998).

Conforme Schwertmann (1988), a maior atividade de Al no sistema pode inibir a formação de goethita em favor da hematita. $O$ processo de substituição isomórfica do Fe pelo Al na estrutura da goethita e a cinética de formação da hematita a partir de gel amorfo de ferridrita são termodinamicamente favorecidos pela rápida desidratação e el evação de temperatura. A taxa de formação de hematita aumenta em até 50 vezes a temperatura de $45^{\circ} \mathrm{C}$, em relação ao processo de formação a ${ }^{28}{ }^{\circ} \mathrm{C}$. Tal variação de temperatura é de ocorrência esperada em crostas microbióticas, que experimentam diariamente extremos térmicos e hídricos.

Desse modo, a oxidação de $\mathrm{Fe}^{2+}$, por influência microbiótica e posterior precipitação na zona de transição entre a crosta e a camada micropedogenizada subjacente, poderia explicar a abundância de micronódulos ferruginosos observada nas fotomicrografias das crostas estudadas (Figura la,b,d,e).

O grau de substituição de Al nos óxidos de Fe presentes foi variável, independentemente da riqueza da fração oxidada ( $\left.\mathrm{Fe}_{\mathrm{D}}\right)$, devendo relacionarse com a proporção de H m/Gt (Schwertmann, 1988), embora essa proporção não tenha sido determinada no presente trabal ho. Os pontos P 5 e P 6 mostraram mai or grau de substituição de Al, sendo o valor em P6 bastante expressivo. Os teores deAl extraído por oxalato $\left(\mathrm{Al}_{\mathrm{O}}\right)$ foram, deforma geral, consideravel mente maiores que aqueles verificados para as formas amorfas de Fe.

De manei ra geral, os teores deAl compl exado pela matéria orgânica (Al-MO) foram mais baixos nos perfis $\mathrm{P} 3$ e $\mathrm{P} 4$, onde o $\mathrm{pH}$ menos ácido favorece a ocorrência deformas cristalinas emenor solubilidade de Al. Nos demais pontos, os valores de $\mathrm{pH}$ devem favorecer altas concentrações deAl trocável, levando ao aumento do processo de substituição isomórfica. No caso de P5, os teores de Al-MO foram mais elevados, sugerindo um processo competitivo entre a substituição isomórfica de Al nos óxidos de Fee a quelação Al-MO. As reações de Al com ácidos orgânicos de baixo peso mol ecular (K wong \& Huang, 1981, e Violante \& Huang, 1985, citados por Huang \& Violante, 1986) e ácidos fúlvicos (Kodama \& Schnitzer, 1980, citado por Huang \& Violante, 1986) podem levar à formação de complexos orgânicos insolúveis dehidróxido deAl, de baixa cristalinidade.

\section{Micropedologia}

As crostas desenvol vidas sobregnai sse mostraram notável diversidade micropedológica (Quadro 7 e Figura 1), ilustrando a complexa interação biogeoquímica mesmo em materiais de natureza geol ógica semel hante. A microestrutura desenvolvida logo abaixo da crosta mostrou tipol ogias desde grãos simples (P4 e P5), microgranular de natureza mais oxídica (P1), até blocos subangulares (P2). Nos saprolitos apédicos (com ausência de estrutura), ocorreu abundante pseudomorfose de biotita em caulinita em todos os pontos, sendo menos pronunciada em P4, ondea biotita encontra-se pouco alterada. As partículas de areia são predominantementequartzoe, secundariamente, pseudomorfos de

Quadro 6. Frações de $\mathrm{Fe}$ e $\mathrm{Al}$ extraídas por oxalato $\left(\mathrm{Fe}_{\mathrm{o}}\right.$ e $\left.\mathrm{Al}_{\mathrm{O}}\right)$, ditionito $\left(\mathrm{Fe}_{\mathrm{D}}\right.$ e $\left.\mathrm{Al}_{\mathrm{D}}\right)$ e solução mista de cloreto de cobre e cloreto de potássio (Fe-MO e Al-MO), na camada intermediária (0-5 mm). Os dados reportam a percentagem de $\mathrm{Fe}_{2} \mathrm{O}_{3}$ e $\mathrm{Al}_{2} \mathrm{O}_{3}$ na fração argila. Fe e Al total refere-se ao teor no solo

\begin{tabular}{|c|c|c|c|c|c|c|c|c|}
\hline Substrato & Feo & $\mathbf{F e}_{\mathbf{D}}$ & Fe total & Fe-MO & Alo & $A I_{D}$ & Al total & Al-MO \\
\hline & 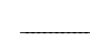 & dag kg-1 & - & $\mathrm{cmol}_{\mathrm{c}} \mathrm{dm}^{-3}$ & 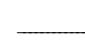 & lag kg- & - & $\mathrm{cmol}_{\mathrm{c}} \mathrm{dm}^{-3}$ \\
\hline Ponto 1 & 0,06 & 7,05 & 5,14 & $\mathrm{Nd}$ & 0,46 & 1,09 & 7,24 & 0,56 \\
\hline Ponto 2 & 0,09 & 6,7 & 2,79 & $\mathrm{Nd}$ & 0,70 & 1,69 & 5,92 & 0,44 \\
\hline Ponto 3 & 0,38 & 7,4 & 5,29 & $\mathrm{Nd}$ & 0,56 & 1,55 & 6,35 & 0,14 \\
\hline Ponto 4 & 0,08 & 4,9 & 5,27 & $\mathrm{Nd}$ & 0,28 & 1,01 & 5,71 & 0,18 \\
\hline Ponto 5 & 0,13 & 11,6 & 8,96 & $\mathrm{Nd}$ & 0,71 & 2,14 & 5,83 & 0,73 \\
\hline Ponto 6 & 0,02 & 4,6 & 3,39 & $\mathrm{Nd}$ & 0,52 & 0,73 & 8,86 & 0,40 \\
\hline
\end{tabular}

$\mathrm{Nd}$ - valores abaixo do limite de detecção. 


\section{Quadro 7. Características micropedológicas selecionadas das crostas estudadas até $3 \mathrm{~cm}$ de profundidade, aproximadamente}

\begin{tabular}{|c|c|c|c|c|c|}
\hline $\begin{array}{c}\text { F eição de } \\
\text { micromorfologia }\end{array}$ & Ponto 1 & Ponto 2 & Ponto 4 & Ponto 5 & Ponto 6 \\
\hline Crosta biológica & $\begin{array}{l}\text { Algas e fungos } \\
\text { filamentosos, } \\
\text { briófitas e líquens; } \\
\text { restos vegetais } \\
\text { de pteridofitas } \\
\text { (rizóides). }\end{array}$ & $\begin{array}{l}\text { Tapete de algas } \\
\text { filamentosas, } \\
\text { raras briófitas, } \\
\text { restos } \\
\text { indecompostos } \\
\text { de rizóides. }\end{array}$ & $\begin{array}{l}\text { Briófitas } \\
\text { abundantes, algas } \\
\text { filamentosas, } \\
\text { líquens foliosos. }\end{array}$ & $\begin{array}{l}\text { Algas } \\
\text { filamentosas, } \\
\text { fungos e raras } \\
\text { briófitas. Tapetes } \\
\text { algais raros. }\end{array}$ & $\begin{array}{l}\text { Tapetes al gais, } \\
\text { fungos, briófitas } \\
\text { elíquens. }\end{array}$ \\
\hline $\begin{array}{l}\text { Microestrutura do } \\
\text { horizonte } \\
\text { micropedogenizado }\end{array}$ & $\begin{array}{l}\text { Microgranular } \\
\text { composta; grãos } \\
\text { de quartzo com } \\
\text { areia fina } \\
\text { predominante. }\end{array}$ & $\begin{array}{l}\text { Blocos } \\
\text { subangulares, com } \\
\text { grãos de quartzo } \\
\text { arestados. }\end{array}$ & Grãos simples. & $\begin{array}{l}\text { Grãos simples } \\
\text { a granular, com } \\
\text { domínio de areia } \\
\text { fina. }\end{array}$ & $\begin{array}{l}\text { Planar com poucos } \\
\text { microagregados. }\end{array}$ \\
\hline $\begin{array}{l}\text { F eições de } \\
\text { alteração mineral } \\
\text { e pedogenética }\end{array}$ & $\begin{array}{l}\text { Concentração } \\
\text { ferruginosa no } \\
\text { contato camada } \\
\text { microped./saprolito; } \\
\text { pseudomorfose de } \\
\text { biotita em caulinita } \\
\text { e feldspato em } \\
\text { gibbsita. }\end{array}$ & $\begin{array}{l}\text { Pseudomorfose } \\
\text { de feldspatos em } \\
\text { gibbisita e } \\
\text { caulinita e de } \\
\text { mica em caulinita; } \\
\text { nódulos } \\
\text { de hemati- } \\
\text { ta/magnetita. }\end{array}$ & $\begin{array}{l}\text { Restos } \\
\text { parcialmente } \\
\text { caulinitizados de } \\
\text { biotita e feldspato; } \\
\text { anfibólios pouco } \\
\text { alterados. }\end{array}$ & $\begin{array}{l}\text { Ferruginização no } \\
\text { contato com o } \\
\text { saprolito; } \\
\text { concentração de } \\
\text { areia fina; planos } \\
\text { de descolamento. }\end{array}$ & $\begin{array}{l}\text { Forte } \\
\text { ferruginização } \\
\text { (hematítica) } \\
\text { envolvendo grãos } \\
\text { de quartzo, com } \\
\text { preenchimento de } \\
\text { poros no saprolito; } \\
\text { pseudomorfose de } \\
\text { feldspatos e micas } \\
\text { em caulinita. }\end{array}$ \\
\hline $\begin{array}{l}\text { Estruturas } \\
\text { associadas }\end{array}$ & $\begin{array}{l}\text { Oxidação biogênica } \\
\text { em micronódulos } \\
\text { hematíticos. }\end{array}$ & $\begin{array}{l}\text { Cutans; } \\
\text { ferruginização } \\
\text { das briófitas; } \\
\text { pedoplasmação } \\
\text { completa; } \\
\text { argiluviação nos } \\
\text { macroporos. }\end{array}$ & $\begin{array}{l}\text { Ferruginização } \\
\text { em restos } \\
\text { orgânicos, bordas } \\
\text { de biotitas e } \\
\text { auréolas } \\
\text { hematíticas nos } \\
\text { anfibólios. }\end{array}$ & $\begin{array}{l}\text { Nódulos } \\
\text { hematíticos e } \\
\text { micropontuações; } \\
\text { pseudomorfose } \\
\text { completa de } \\
\text { biotita em } \\
\text { caulinita; raros } \\
\text { cutans no } \\
\text { saprolito. }\end{array}$ & $\begin{array}{l}\text { Micronódulos } \\
\text { hematíticos } \\
\text { biogênicos; } \\
\text { bolsões orgânicos } \\
\text { indeterminados. }\end{array}$ \\
\hline
\end{tabular}

Nota: o ponto 3 não foi estudado em seção fina.

bi otita caulinitizados degrandes dimensões (silteaté areia grossa). Em P4, existem também grãos de anfibólio pouco alterados, com uma auréola de alteração ferruginosa. Na crosta e na camada micropedogenizada, há ocorrência generalizada de micronódulos e micropontuações ferruginosas, que mostram uma forte participação microbiótica em sua gênese (Schwertmann, 1988; Fischer, 1988).

\section{Desenvolvimento de algas em blocos indefor- mados}

Algas fotoautotróficas como as cianobactérias, organismos procariotos, morfologicamente e fisiol ogi camente semel hantes às bactérias (Albrecht, 1999), sintetizam os metabol itos essenciais a partir do $\mathrm{CO}_{2}$ atmosférico, em presença de energia luminosa. O procedimento utilizado, com o emprego da solução Bold Basal Medium, permitiu o desenvolvimento de uma população diversificada de algas já na terceira semana de incubação, com o predomínio das espécies unicelulares sobre as espécies filamentosas em praticamente todos os meios de cultura.

As algas filamentosas, apresentando talo multisseriado, algumas vezes mostrando a junção de indivíduos unicelulares em forma de bastonetes, abundantes nos meios de cultura referentes aos encrostamentos de P4 eP5 (Figura 1g,h,i ), têm papel importante no processo de agregação, em virtude do invólucro de partículas minerais e da liberação de compostos orgânicos extracel ulares, pol issacarídeos (Figura 2a,b), os quais aumentam a estabilidade dos agregados em água (Chenu, 1993).

Foram observadas também associações de colônias de espécies unicelulares com algas filamentosas (Figuras 1f), colônias de indivíduos unicelulares com arranjo em pacote ou tendendo à formação defilamentos, ediatomáceas, aproveitandose de microambiente favorável propiciado pelas cianobactérias, pioneiras no processo de sucessão ecológica.

Em laboratório, as al gas, incubadas em saprolitos sem evidente colonização biológica, mostraram rápido desenvolvimento, tanto no material arenoso ( $<15 \%$ de argila) quanto no material mais argiloso, observável num período de duas semanas. A observação visual durante o experimento, entretanto, permitiu identificar um processo de estruturação mais expressivo no substrato arenoso em relação ao argiloso, o que é consistente com 


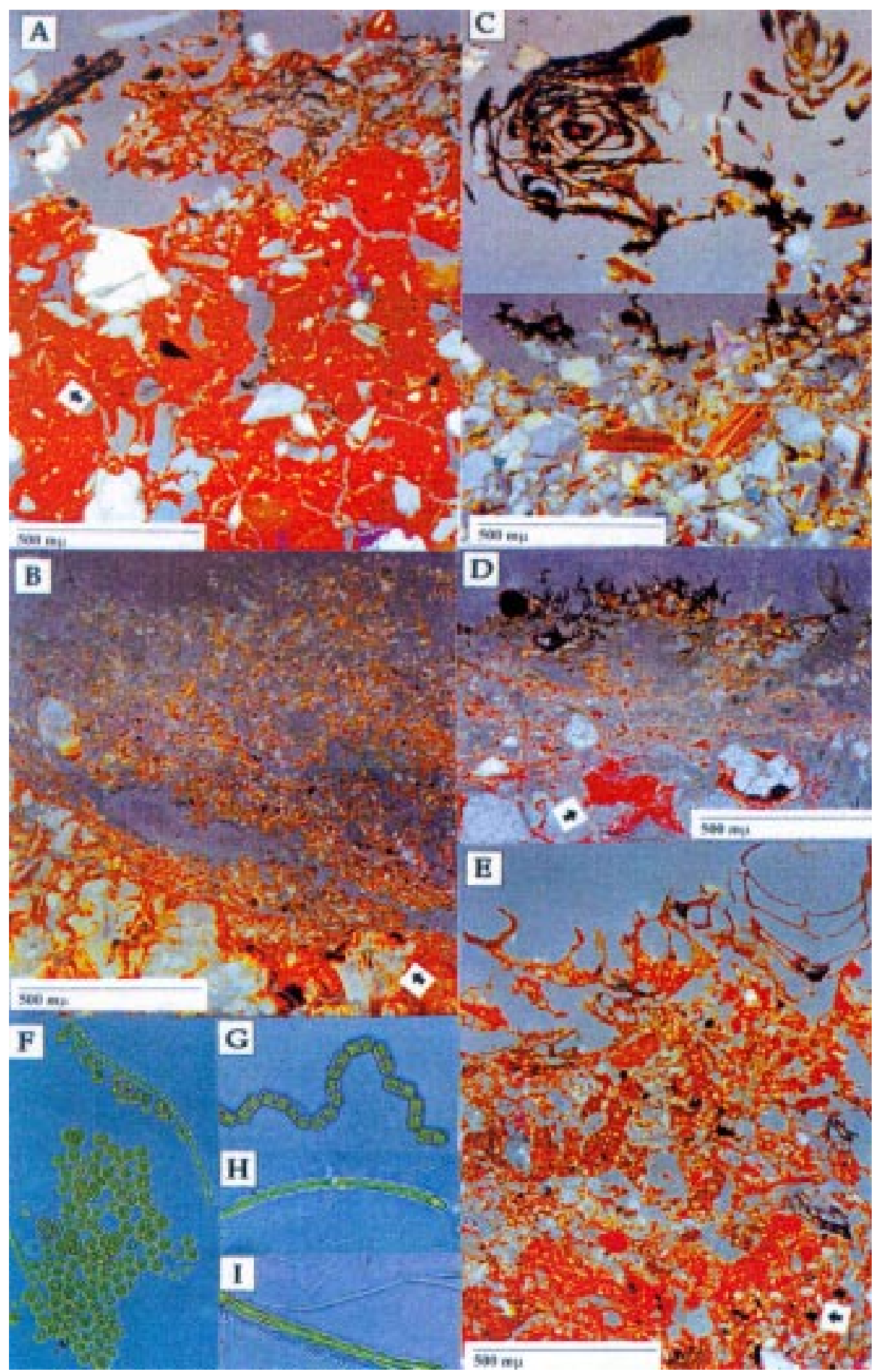

Figura 1. Fotomicrografias das crostas biológicas em A, B, C, D e E; e das cianobactérias desenvolvidas em meio de cultura, em F, G, H e I. A) E strutura em blocos com inci piente desenvolvimento de cutans de tensão (zonas amareladas, seta) na superfície de agregados, notando-se ferruginização na parte superior da crosta (P2); B) crosta biológica bem desenvolvida, em P5, com abundantes micropontuações biogênicas e pseudomorfose de biotita em caulinita (seta), notando-se ferrugi nização acentuada na base da camada micropedogenizada; C) Briófitas em processo de decomposição sobre camada delgada, micropedogenizada em grãos simples (P4); D) Crosta de líquens e briófitas sobre tapete de algas decompostas, em P6, notando-se abundância de pontuações ferruginosas e zonas de plasma ferrugi noso avermelhado (seta) no saprolito; E) Líquens foliosos, em P 1, recobrindo horizonte micropedogenizado, com abundantes micronódulos ferruginosos (seta); F) Associações de colônias de algas unicelulares com algas filamentosas (200X); G) J unção de indivíduos unicelulares em forma de bastonetes formando filamentos multisseriados (400X); H) Algas filamentosas, apresentando talo multisseriado (400X); I) Filamento de alga com ramificações (400X). 


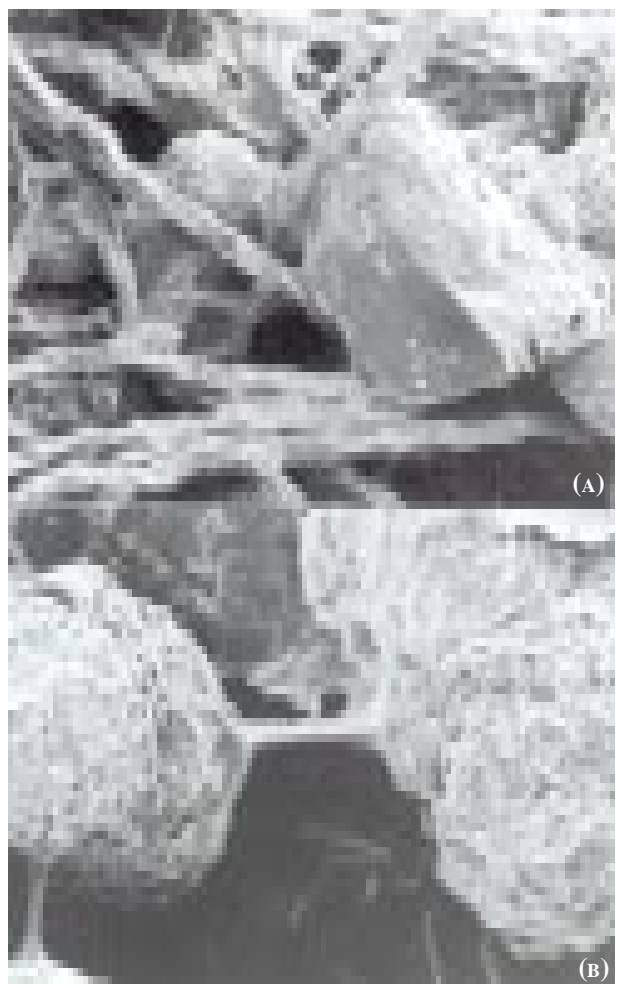

Figura 2. Fotomicrografias em ME V, ilustrando aspectos da interação biota/saprolito nas crostas de P5. A) Cianobactérias filamentosas envolvendo grãos de quartzo e agregados organoargilosos com abundante muco polissacarídeo (200X); B) Agregados minerais subesféricos ligados por exsudatos de polissacarídeos, com importante papel na sua estabilidade (500X).

observações de Oades (1993), segundo as quais as influências abióticas em solos argilosos, como os ciclos de umedecimento e secagem, teriam efeitos mais importantes.

\section{CONCLUSÕES}

1. As populações de microrganismos, associadas à microflora, foram responsáveis pela estruturação incipiente dos saprolitos gnáissicos subjacentes e ainda pela efetiva ciclagem de nutrientes, propiciando a formação de um meio pedogenizado favorável ao crescimento de plantas superiores. Esta interação organomineral envolveu, de início, a fixação de $\mathrm{C}$ orgânico, a partir do $\mathrm{CO}_{2}$ atmosférico, por algas cianobactérias.

2. Líquens, resultantes da associação mutualística de fungos (função protetora) ecianobactérias (função fotossintetizante e fixação de N), cresceram sobre a superfície mineral, em sucessão, enquanto as briófitas utilizaram os nutrientes liberados neste processo na produção de biomassa. N este ponto, a crosta estava capacitada a abrigar pteridófitas e as primeiras plantas superior es com raízes, em decorrência da crescente estabilidade de agregados promovida pela abundante produção de polissacarídeos.

3. As espécies dealgas isol adas em meio decultura e inoculadas em ambiente controlado mostraramse eficientes em colonizar os saprolitos, após duas semanas de incubação. Os resultados evidenciaram a possibilidade do uso de inóculo de cianobactérias na estabilização detaludes e a recuperação deáreas degradadas, como técnica complementar baseada na aceleração do processo natural de sucessão ecol ógica.

\section{AGRADECIMENTOS}

Os autores agradecem ao CNPq, pela concessão da bolsa de estudo durante o desenvol vimento deste trabalho.

\section{LITE RATURA CITADA}

ALBRECHT, S.L. Eukaryotic algae and cyanobacteria. In: SYLVIA, D.M.; FUHRMANN, J J .; HARTEL, P.G. \& ZUBERER, D.A., eds. Principles and applications of soil microbiology. U pper Saddle River, Prentice Hall, 1999. p.94113.

ARTUR, A.C. \& WERNICK, E. Model os geotectônicos aplicados ao Pré-Cambriano Superior no NE do Estado de São Paulo e áreas adjacentes do estado de Minas Gerais: uma discussão. Geociências, 12:155-185, 1993.

BARKER, W.W.; WELCH, S.A. \& BANFIELD, J .F. Biogeochemical weathering of silicate minerals. In: BANFIELD, J.F. \& NEALSON, K.H., eds. Reviews in mineralogy. geomicrobiology: interactions between microbes and minerals. Miner. Soc. Am., 35:391-428, 1998.

BRASIL. Ministério das Minas e Energia. Programa de levantamentos geológicos básicos do Brasil. Rio Espera. Fol ha SF 23-X-B-IV, Estado de Minas Gerais. Escala 1:100 000. Texto explicativo. Brasília, DNPM/CPRM, 1991, 200p.

BRE MNER, J .M. Nitrogen-Total. I n: SPARKS, D.L., ed. Methods of soil analysis. Part 3. Madison, American Society of Agronomy, 1996. p. 1085-1121. (SSSA Book Series: 5)

CHENU, C. Clay - or sand - polysaccharide associations as models for the interface between micro-organisms and soil: water related properties and microstructure. Geoderma, 56:143-156, 1993.

COFFIN, D.E. A method for the determination of free iron in soils and clays. Can. J. Soil Sci., 43:7-17, 1963.

EHRLICH, H.L. Geomicrobiology. 3.ed. New York, Marcel Dekker, 1996. p.108-113. 
EMPRESA BRASILEIRA DE PESQUISA AGROPECUÁRIA EMBRAPA. Manual de métodos de análise de solo. 2.ed. Rio de J aneiro, 1997. 212p.

FISCHER, W.R. Microbiological reactions of iron in soils. In: STUCKI, J.W.; GOODMAN, B.A. \& SCHWERTMANN, U., eds. Iron in soils and clay minerals. Dordrecht, D. Reidel Publishing Company, 1988. p.715-748. (NATO Advanced Study C, Mathematical and physical sciences, v. 217)

FITZPATRICK, E.A. Soil microscopy and micromorphology. Chichester, J ohn Wiley, 1993. 304p.

GORBUSHINA, A.A. \& KRUMBEIN, W.E. Subaerial microbial mats and their effects on soil and rock. In: RIDING, R.E. \& AWRAMIK, S.M., eds. Microbial sediments. Berlin, Springer-Verlag, 2000. p.161-170.

HUANG, P.M. \& VIOLANTE, A. Influence of organic acids on crystallization and surface properties of precipitation products of aluminium. In: HUANG, P.M. \& SCHNITZER, $M$., eds. Interactions of soil minerals with natural organics and microbes. Madison, Soil Science Society of America, 1986. p.159-221. (SSSA Special Publication, 17)

LONGTON, R.E. The role of bryophytes and lichens in terrestrial ecosystems. In: BATES, J.W. \& FARMER, A.M., eds. Bryophytes and lichens in a changing environment. Oxford, Clarendon Press, 1992. p.32-76.

McKEAGUE,J.A \& DAY,J .H. Dithionite and oxalate- extractable $\mathrm{Fe}$ and $\mathrm{Al}$ as aids in differentiating various classes of soils. Can. J. Soil Sci., 46:13-22, 1966.

MEHRA, O.P. \& J ACKSON, M.L. I ron oxide removal from soils and clays by a dithionite-citrate system buffered with sodium bicarbonate. New York, Pergamon Press, 1960. p.317-327.

METTING, F.B. Algae and cyanobacteria. In: PAGE, A.L., ed. Methods of soil analysis. Part 2. Madison, Soil Science Society of America, 1994. p.427-458.

NELSON, D.W. \& SOMMERS, L.E. Total carbon, organic carbon, and organic matter. In: PAGE, A.L., ed. Methods of soil analysis. Part 2. Madison, Soil Science Society of America, 1982. p.539-579.

NEUMAN, C.M.; MAXWELL, C.D. \& BOULTON, J.W. Wind transport of sand surfaces crusted with photoautotrophic microorganisms. Catena, 27:229-247, 1996.

OADES, J.M. Soil organic matter and structural stability: mechanisms and implications for management. In: TINSLEY, J \& \& DARBYSHIRE, J .F., eds. Biological processes and soil fertility. The Hague, Martinus Nighoff / Dr. W. J unk Publishers, 1984. p.319-337. (Developments in Plant and Soil Sciences, 11)

OADES, J.M. The role of biology in the formation, stabilization and degradation of soil structure. Geoderma, 53:377-400, 1993.
PÉREZ, F.L. Microbiotic crusts in the high equatorial Andes, and their influence on paramo soils. Catena, 31:173-198, 1997.

ROBERT, M. \& BERTHELIN, J . Role of biological and biochemical factors in soil mineral weathering. In: HUANG, P.M. \& SCHNITZER, M., eds. Interactions of soil minerals with natural organics and microbes. Madison, Soil Science Society of America, 1986. p.453-495. (SSSA Special Publication, 17)

SCHWERTMANN, U. Occurrence and formation of iron oxides in various pedoenvironments. In: STUCKI, J.W.; GOODMAN, B.A. \& SCHWERTMANN, U., eds. I ron in soils and clay minerals. Dordrecht, D. Reidel Publishing Company, 1988. p.267-308. (NATO Advanced Study C, Mathematical and Physical Sciences, v. 217)

SHEN, S.; PEPPER, G.E.; HASSETT, J .J . \& STUCKI, J .W. Acidity and aluminum toxicity caused by iron oxidation around anode bars. Soil Sci., 163:657-664, 1998.

SILVER, M.; EHRLICH, H.L. \& IVARSON, K.C. Soil mineral transformation mediated by soil microbes. In: HUANG, P.M. \& SCHNITZER, M., eds. Interactions of soil minerals with natural organics and microbes. Madison, Soil Science Society of America, 1986. p.497-519. (SSSA Special Publication, 17)

SOON, Y.K. Fractionation of extractable aluminium in acid soil: a review and a proposed procedure. Comm. Soil Sci. Plant Anal., 24:1683-1708, 1993.

STONE, A.T. Reactions of extracellular organic ligands with dissolved metal ions and mineral surfaces. In: BANFIELD, J.F. \& NEALSON, K.H., eds. Reviews in mineralogy. geomicrobiology: interactions between microbes and minerals. Miner. Soc. Am., 35:309-344, 1998.

TAN, K.H. The release of silicon, aluminium, and potassium during decomposition of soil minerals by humic acid. Soil Sci., 129:5-11, 1980.

TEDESCO, M.J .; WOLKWEISS, S.J . \& BOHNEN, H. Análise de solo, planta e outros materiais. Porto Alegre, Universidade Federal do Rio Grande doSul, 1985. 156p. (Bol etim Técnico, 5)

TISDALL, J .M. \& OADES, J .M. Organic matter and water-stable aggregates in soils. J. Soil Sci., 33:141-163, 1982.

WHITTIG, L.D. \& ALLARDICE, W.R. X-ray diffraction techniques. In: KLUTE, A., ed. Methods of soil analysis. Part 1. Madison, American Society of Agronomy / Soil Science Society of America, 1986. p.331-362.

ZIMMERMAN, W.J . Microbial biotechnology and applications in agriculture. In: METTING, F.B., ed. Soil microbial ecology. Applications in agricultural and environmental management. New York, Marcel Dekker, 1993. p.457-479. 
E.S. TRINDADE et al.

R. Bras. Ci. Solo, 25:849-861, 2001 